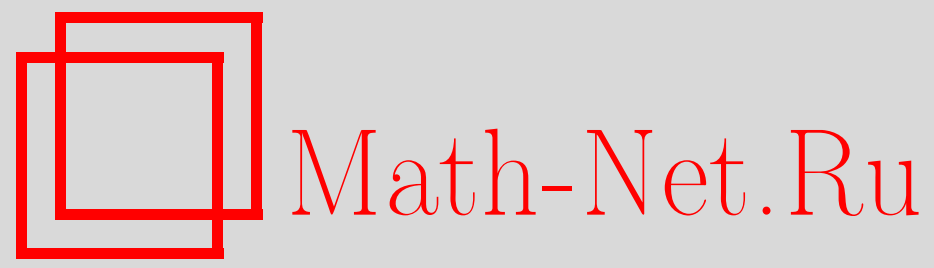

А. А. Серов, Оценки объемов окрестностей двоичных кодов в терминах их весовых спектров, Матем. вопр. криптогр., 2013, том 4, выпуск 2, 17-42

DOI: https://doi.org/10.4213/mvk79

Использование Общероссийского математического портала Math-Net.Ru подразумевает, что вы прочитали и согласны с пользовательским соглашением

http://www . mathnet.ru/rus/agreement

Параметры загрузки:

IP : 54.237 .59 .107

26 апреля 2023 г., 12:45:15 
УДК: 519.724

\title{
Оценки объемов окрестностей двоичных кодов в терминах их весовых спектров
}

\author{
A. А. Серов \\ Математический институт им. В. А. Стеклова РАН, Москва
}

Получено 20.IV.2012

В работе получены двусторонние оценки числа элементов, принадлежащих $r$-окрестности кода, в терминах спектра расстояний между кодовыми словами. Оценки конкретизированы для кодов Рида-Мюллера первого и второго порядков. Кроме того, приводится обзор кодов, для которых спектр расстояний известен, и отмечены некоторые приложения теории кодов к криптографии.

Ключевые слова: двоичные коды, объемы окрестностей, коды РидаМюллера, спектр расстояний, формула включения-исключения

Estimates of the neighborhood volumes of binary codes via their weight spectra

\author{
A. A. Serov \\ Steklov Mathematical Institute of RAS, Moscow
}

Abstract. We obtain two-sided estimates for the number of elements in the $r$-neighborhood of a code via the spectrum of distances between codewords. For the first and second order Reed-Muller codes the estimates are more explicit. A short review of codes with known distance spectrum is given and some applications of the coding theory to cryptography are discussed.

Key words: binary codes, neighborhood volumes, Reed-Muller codes, distance spectrum, inclusion-exclusion formula

Citation: Mathematical Aspects of Cryptography, 2013, vol. 4, no. 2, pp. 17-42 (Russian). 


\section{$\S 1$. Основные понятия и обозначения}

Пусть $X=\left\{a_{0}, \ldots, a_{q-1}\right\}$ - конечное $q$-элементное множество. Множество $X^{n}$ состоит из всех $n$-мерных векторов с координатами из множества $X$. Очевидно, $\left|X^{n}\right|=q^{n}$. На множестве $X^{n}$ вводится метрика Хеммингa: расстояние $\mathrm{d}(x, y)$ между векторами $x, y \in X^{n}$ считается равным числу позиций, в которых они различаются.

Произвольное подмножество элементов метрического пространства $X^{n}$ называется кодом $\mathcal{C}$ (см., например, [45]), а элементы кода - кодовыми словами. Число $n$ называют длиной кодовых слов и самого кода.

Расстоянием от произвольного $x \in X^{n}$ до кода $\mathcal{C}$ называется величина

$$
\mathrm{d}(x, \mathcal{C})=\min _{y \in \mathcal{C}} \mathrm{d}(x, y)
$$

Кодовым расстоянием $d=\mathrm{d}(\mathcal{C})$ кода $\mathcal{C}$ называется минимальное расстояние между двумя различными элементами кода $\mathcal{C}$ :

$$
\mathrm{d}(\mathcal{C})=\min _{a, b \in \mathcal{C}: a \neq b} \mathrm{~d}(a, b)
$$

Для удобства задания кода обычно в качестве $X$ рассматривается множество с какой-либо алгебраической структурой, например конечное поле или конечное кольцо.

Если $X$ - конечное поле $\mathbb{F}_{q}, q=p^{l}$, где $p$ - простое число, то $X^{n}$ можно рассматривать как $n$-мерное пространство над полем $\mathbb{F}_{q}$. Будем обозначать его через $\mathbb{F}_{q}^{n}$.

Если в множестве $X$ есть нулевой элемент 0 , то в $X^{n}$ выделяется нулевой вектор (все координаты которого равны 0), и расстояние от вектора $x \in X^{n}$ до нулевого вектора называется весом Хемминга (или просто весом) $\mathrm{wt}(x)$ вектора $x$.

Линейное подпространство пространства $\mathbb{F}_{q}^{n}$ называется линейным кодом. Кодовое расстояние линейного кода $\mathcal{C} \subset \mathbb{F}_{q}^{n}$ равно минимальному весу ненулевого вектора в линейном подпространстве $\mathcal{C}$ :

$$
d(\mathcal{C})=\min _{a \in \mathcal{C} \backslash\{0\}} \operatorname{wt}(a)
$$

Через $k=\operatorname{dim} \mathcal{C}$ будем обозначать размерность линейного кода $\mathcal{C} \subset$ $\subset \mathbb{F}_{q}^{n}$. Код длины $n$ размерности $k$ с кодовым расстоянием $d$ будем называть $[n, k, d]$-кодом. 
Пусть $\omega=\left\{\omega_{1}, \ldots, \omega_{k}\right\}-$ базис пространства $\mathcal{C}$. Матрицу $A=A(\mathcal{C})$ размера $k \times n$, строками которой являются векторы $\left\{\omega_{1}, \ldots, \omega_{k}\right\}$, принято называть порождающей матрищей кода $\mathcal{C}$. Любой вектор $x \in \mathcal{C}$ можно представить в виде

$$
x=z A,
$$

где $z$ - вектор пространства $\mathbb{F}_{q}^{k}$.

Линейный код можно задавать также проверочной матрицей Н размера $(n-k) \times n \mathrm{c} n-k$ линейно независимыми строками, т. е. как множество всех таких векторов $x=\left(x_{1}, \ldots, x_{n}\right)$, что $\mathbf{H} x^{T}=0$.

Пусть $\mathcal{C}$ - линейный код с порождающей матрицей $\boldsymbol{A}$ и проверочной матрицей $\mathbf{H}$. Код $\mathcal{C}^{\perp}$ с порождающей матрицей $\mathbf{H}$ и проверочной матрицей $\boldsymbol{A}$ называется дуальным кодом для $\mathcal{C}$.

Многочлены $A(z)=\sum_{i=0}^{n} A_{i} z^{i}$ и $B(z)=\sum_{i=0}^{n} B_{i} z^{i}$, где $A_{i}$ и $B_{i}-$ числа кодовых слов веса $i$ в кодах $\mathcal{C}$ и $\mathcal{C}^{\perp}$ соответственно, называются весовыми энумераторами кодов $\mathcal{C}$ и $\mathcal{C}^{\perp}$.

Для весовых энумераторов $A(z)$ и $B(z)$ любого линейного кода $\mathcal{C}$ и дуального кода $\mathcal{C}^{\perp}$ справедливо тождество Мак-Вильямс ( [42]):

$$
B(z)=\frac{1}{|\mathcal{C}|}(1+z)^{n} A\left(\frac{1-z}{1+z}\right) .
$$

Для нелинейного кода нет естественного определения дуального кода. Однако каждому нелинейному коду соответствует весовой энумератор $A(z)$. Существуют примеры нелинейных кодов, весовые энумераторы которых связаны тождеством Мак-Вильямс; такими являются, например, коды Препараты [28] и Кердока [13].

\section{§2. Оценки объемов окрестностей двоичных кодов}

В этом параграфе рассматриваются двоичные коды $\mathcal{C}$ длины $n$ с минимальным расстоянием $d$. Теорема 1 относится к двоичным дистаниионноинвариантным кодам, т. е. к таким подмножествам $\mathcal{C}$ пространства $\mathbb{F}_{2}^{n}$, что для любого $x \in \mathcal{C}$ совокупность расстояний Хемминга $\{\rho(x, y), y \in \mathcal{C}\}$ не зависит от $x$. Частными случаями дистанционно-инвариантных кодов являются описанные в 33 коды Рида-Мюллера, а также совершенные коды (см., например, $[41,48])$. Теорема 2 относится к произвольным двоичным кодам.

Окрестности кодовых слов можно представлять себе, как в [37], шарами целочисленного радиуса $r$. Если при $r=a, a \in \mathbb{N}$, шары не пересекаются, а при $r=a+1$ пересекаются, то $a$ называется радиусом сферической упаковки 
кода. Минимальное значение $b$ радиуса $r$, при котором каждая точка $\mathbb{F}_{2}^{n}$ попадает в $b$-окрестность хотя бы одного кодового слова, называется радиусом покрытия кода.

Нахождение количеств векторов $v \in \mathbb{F}_{2}^{n}$, попадающих в $r$-окрестность кода $\mathcal{C}$

$$
\mathbb{F}_{2}(\mathcal{C}, r)=\left\{v \in \mathbb{F}_{2}^{n}: \mathrm{d}(v, \mathcal{C}) \leqslant r\right\},
$$

представляет практический интерес. При $r \leqslant a$ это количество равно произведению числа векторов в шаре радиуса $r$ и числа кодовых слов, при $r \geqslant b$ оно равно $2^{n}$. В настоящем параграфе для чисел векторов в $r$-окрестностях кодов при $a<r<b$ с помощью формул включения-исключения получены двусторонние неравенства в терминах весового спектра кода.

Пространство $\mathbb{F}_{2}^{n}$ разбивается на слои $\mathbb{F}_{2}^{n}(i)=\left\{x \in \mathbb{F}_{2}^{n}: \operatorname{wt}(x)=i\right\}$ :

$$
\mathbb{F}_{2}^{n}=\bigcup_{i=0}^{n} \mathbb{F}_{2}^{n}(i)
$$

Положим

$$
N_{n}^{(2)}(i, r) \stackrel{\text { def }}{=}\left|\left\{x \in \mathbb{F}_{2}^{n}: \max \{\mathrm{d}(x, 0), \mathrm{d}(x, c)\} \leqslant r, \operatorname{wt}(c)=i\right\}\right| .
$$

Корректность этого определения следует из того, что при любом фиксированном значении $i$ правая часть не зависит от вектора $c, \operatorname{wt}(c)=i$, так как любой вектор веса $i$ можно перевести в любой другой вектор такого же веса перенумерацией координат, а она не изменяет веса векторов и расстояния Хемминга между ними.

Теорема 1. Пусть $\mathcal{C} \subset \mathbb{F}_{2}^{n}-$ двоичный дистанщионно-инвариантный код длины п с минимальным расстоянием $d u$

$$
W_{i}=\mathbb{F}_{2}^{n}(i) \cap \mathcal{C}, \quad i \in\{0,1, \ldots, n\},
$$

- множества кодовых слов одинакового веса, $\left\{\left|W_{i}\right|\right\}_{i=0}^{n}-$ весовой спектр кода С. Тогда

$$
\left|\mathbb{F}_{2}(\mathcal{C}, r)\right|=(1-q(n, r))|\mathcal{C}| \sum_{m=0}^{r} C_{n}^{m},
$$

где $q(n, r)=0$ при $0 \leqslant r<d / 2 u$

$$
0 \leqslant q(n, r) \leqslant\left(2 \sum_{m=0}^{r} C_{n}^{m}\right)^{-1} \sum_{i=1}^{n}\left|W_{i}\right| N_{n}^{(2)}(i, r), \quad d / 2 \leqslant r \leqslant n .
$$


Ряд явных оценок для $\sum_{m=0}^{r} C_{n}^{m}$ и $N_{n}^{(2)}(i, r)$ приведен ниже в утверждениях 1 и 2.

Теорема 2. Пусть $\mathcal{C} \subset \mathbb{F}_{2}^{n}-$ двоичный код длины $n$ с минимальным расстоянием d,

$$
W_{i}^{(2)}=\left\{\left(c_{1}, c_{2}\right): c_{1}, c_{2} \in \mathcal{C}, \mathrm{d}\left(c_{1}, c_{2}\right)=i\right\}, \quad i \in\{0,1, \ldots, n\},
$$

- множества упорядоченных пар кодовых слов, расстояние между которыми принимает заданное значение, $и \mathbb{F}_{2}(\mathcal{C}, r)=\left\{x \in \mathbb{F}_{2}^{n}: \mathrm{d}(x, \mathcal{C}) \leqslant r\right\}-$ r-окрестность кода $\mathcal{C}$. Тогда

$$
\left|\mathbb{F}_{2}(\mathcal{C}, r)\right|=(1-q(n, r))|\mathcal{C}| \sum_{m=0}^{r} C_{n}^{m}
$$

где $q(n, r)=0$ при $0 \leqslant r<d / 2 u$

$$
0 \leqslant q(n, r) \leqslant\left(2|\mathcal{C}| \sum_{m=0}^{r} C_{n}^{m}\right)^{-1} \sum_{i=1}^{n}\left|W_{i}^{(2)}\right| N_{n}^{(2)}(i, r), \quad d / 2 \leqslant r \leqslant n .
$$

Доказательство теоремы 1 . Пусть $\mathcal{C}=\left\{c_{1}, c_{2}, \ldots, c_{|\mathcal{C}|}\right\}$. Так как

$$
\mathbb{F}_{2}(\mathcal{C}, r)=\bigcup_{i=1}^{|\mathcal{C}|}\left\{x \in \mathbb{F}_{2}^{n}: \mathrm{d}\left(x, c_{i}\right) \leqslant r\right\}
$$

то согласно формуле включения-исключения [47]

$$
\begin{gathered}
\sum_{u=1}^{|\mathcal{C}|}\left|\left\{x \in \mathbb{F}_{2}^{n}: \mathrm{d}\left(x, c_{u}\right) \leqslant r\right\}\right|- \\
\left.-\sum_{1 \leqslant u<v \leqslant|\mathcal{C}|} \mid x \in \mathbb{F}_{2}^{n}: \max \left\{\mathrm{d}\left(x, c_{u}\right), \mathrm{d}\left(x, c_{v}\right)\right\} \leqslant r\right\} \mid \leqslant \\
\leqslant \mathbb{F}_{2}(\mathcal{C}, r) \leqslant \sum_{u=1}^{|\mathcal{C}|}\left|\left\{x \in \mathbb{F}_{2}^{n}: \mathrm{d}\left(x, c_{u}\right) \leqslant r\right\}\right| .
\end{gathered}
$$

Очевидно, при каждом $u \in\{1,2, \ldots,|\mathcal{C}|\}$

$$
\left|\left\{x \in \mathbb{F}_{2}^{n}: \mathrm{d}\left(x, c_{u}\right) \leqslant r\right\}\right|=\sum_{m=0}^{r} C_{n}^{m} .
$$

2013. T. 4. № 2. C. 17-42 
Число таких слагаемых в (1) равно $|\mathcal{C}|$. Поэтому

$$
\left|\mathbb{F}_{2}(\mathcal{C}, r)\right| \leqslant \sum_{1 \leqslant u \leqslant|\mathcal{C}|}\left|\left\{x \in \mathbb{F}_{2}^{n}: \mathrm{d}\left(x, c_{u}\right) \leqslant r\right\}\right|=|\mathcal{C}| \sum_{m=0}^{r} C_{n}^{m},
$$

тем самым доказана оценка сверху.

Для любых кодовых слов $c_{u}, c_{v} \in \mathcal{C}, u, v \in\{1,2, \ldots,|\mathcal{C}|\}, u \neq v$,

$$
\begin{aligned}
\mid\left\{x \in \mathbb{F}_{2}^{n}:\right. & \left.\max \left\{\mathrm{d}\left(x, c_{u}\right), \mathrm{d}\left(x, c_{v}\right)\right\} \leqslant r\right\} \mid= \\
& =\left|\left\{x \in \mathbb{F}_{2}^{n}: \max \left\{\mathrm{d}\left(x \oplus c_{u}, c_{u} \oplus c_{u}\right), \mathrm{d}\left(x \oplus c_{u}, c_{v} \oplus c_{u}\right)\right\} \leqslant r\right\}\right| .
\end{aligned}
$$

Число таких упорядоченных пар $\left(c_{u}, c_{v}\right)$ кодовых слов кода $\mathcal{C}$, что $\mathrm{d}\left(c_{u}, c_{v}\right)=i$, равно $|\mathcal{C}| \cdot\left|W_{i}\right|$, а неупорядоченных - в два раза меньше. Отсюда следует, что для каждого фиксированного $r \leqslant n$ вторая сумма в левой части (1) совпадает с

$$
\begin{gathered}
\frac{|\mathcal{C}|}{2} \sum_{i=1}^{n}\left|W_{i}\right|\left|\left\{x \in \mathbb{F}_{2}^{n}: \max \{\mathrm{d}(x, 0), \mathrm{d}(x, b)\} \leqslant r, \operatorname{wt}(b)=i\right\}\right|= \\
=\frac{|\mathcal{C}|}{2} \sum_{i=1}^{n}\left|W_{i}\right| N_{n}^{(2)}(i, r) .
\end{gathered}
$$

Поэтому

$$
\left(1-q^{*}(n, r)\right)|\mathcal{C}| \sum_{m=0}^{r} C_{n}^{m} \leqslant\left|\mathbb{F}_{2}(\mathcal{C}, r)\right| \leqslant|\mathcal{C}| \sum_{m=0}^{r} C_{n}^{m},
$$

если

$$
q^{*}(n, r)=\frac{1}{2} \sum_{i=1}^{n}\left|W_{i}\right| N_{n}^{(2)}(i, r) / \sum_{m=0}^{r} C_{n}^{m} .
$$

Доказательство теоремы 2 отличается от доказательства теоремы 1 лишь тем, что для каждого фиксированного $r \leqslant n$ вторая сумма в левой части (1) совпадает с

$$
\begin{aligned}
\frac{1}{2} \sum_{i=1}^{n}\left|W_{i}^{(2)}\right| \cdot \mid\{x \in & \left.\mathbb{F}_{2}^{n}: \max \{\mathrm{d}(x, 0), \mathrm{d}(x, b)\} \leqslant r, \mathrm{wt}(b)=i\right\} \mid= \\
& =\frac{1}{2} \sum_{i=1}^{n}\left|W_{i}^{(2)}\right| N_{n}^{(2)}(i, r) .
\end{aligned}
$$


Тогда согласно формуле (1)

$$
|\mathcal{C}| \sum_{m=0}^{r} C_{n}^{m}-\frac{1}{2} \sum_{i=1}^{n}\left|W_{i}^{(2)}\right| N_{n}^{(2)}(i, r) \leqslant\left|\mathbb{F}_{2}(\mathcal{C}, r)\right| \leqslant|\mathcal{C}| \sum_{m=0}^{r} C_{n}^{m},
$$

поэтому при

$$
q(n, r)=\left(2|\mathcal{C}| \sum_{m=0}^{r} C_{n}^{m}\right)^{-1} \sum_{i=1}^{n}\left|W_{i}^{(2)}\right| N_{n}^{(2)}(i, r)
$$

получаем утверждения теоремы 2.

Утверждение 1. При $r \leqslant n / 2$ справедливы оценки

$$
2^{n} \Phi\left(-\sqrt{n V\left(1-\frac{2 r}{n}\right)}\right) \leqslant \sum_{m=0}^{r} C_{n}^{m} \leqslant 2^{n} \Phi\left(-\sqrt{n V\left(1-\frac{2(r+1)}{n}\right)}\right),
$$

где $\Phi(x)=\frac{1}{\sqrt{2 \pi}} \int_{-\infty}^{x} e^{-u^{2} / 2} d u-$ функция стандартного нормального распределения и при $|z|<1$

$$
V(z)=(1-z) \ln (1-z)+(1+z) \ln (1+z)=\sum_{s=1}^{\infty} \frac{z^{2 s}}{s(2 s-1)} \geqslant z^{2} .
$$

Доказательство. Будем обозначать через $X_{n, p}$ случайную величину, имеющую биномиальное распределение с параметрами $(n, p)$. Неравенство (2) следует из тождеств

$$
\begin{gathered}
\sum_{m=0}^{r} C_{n}^{m}=\sum_{m=n-r}^{n} C_{n}^{m}= \\
=2^{n} \mathbf{P}\left\{X_{n, 1 / 2} \geqslant n-r\right\}=2^{n}\left(1-\mathbf{P}\left\{X_{n, 1 / 2} \leqslant n-r-1\right\}\right)
\end{gathered}
$$

и приведенных в [39, 40] переформулировок некоторых результатов статьи [2] о хвостах биномиального распределения.

Стандартные неравенства для хвостов функции нормального распределения можно найти в [47], гл. 7, §1:

$$
\frac{e^{-x^{2} / 2}}{\sqrt{2 \pi}}\left(\frac{1}{x}-\frac{1}{x^{3}}\right)<\Phi(-x)=1-\Phi(x)<\frac{e^{-x^{2} / 2}}{\sqrt{2 \pi}} \frac{1}{x}, \quad x>0,
$$

а также в справочнике [36], пп. 26.2.24-26.2.25.

2013. T. 4. № 2. C. 17-42 
Утверждение 2. Если $0 \leqslant r \leqslant[n / 2] u 0 \leqslant i \leqslant n$, то

$$
N_{n}^{(2)}(i, r) \leqslant\left\{\begin{array}{l}
C_{i}^{i / 2} C_{n-i}^{r-i / 2} \frac{1+q_{i}}{\left(1-q_{i}\right)^{2}} \text { при четном } i ; \\
C_{i}^{[i / 2]} C_{n-i}^{r-[i / 2]} \frac{2}{\left(1-q_{i}\right)^{2}} \text { при нечетном } i,
\end{array}\right.
$$

где $q_{i}=\frac{r-[i / 2]}{n-i+[i / 2]-r+1} \leqslant q=\frac{r}{n-r+1}$.

Доказательство. При фиксированном $b=\left(b_{1}, \ldots, b_{n}\right) \in \mathbb{F}_{2}^{n}$ сопоставим каждому вектору $a=\left(a_{1}, \ldots, a_{n}\right) \in \mathbb{F}_{2}^{n}$ величины

$$
\begin{aligned}
& m_{0}=\left|\left\{i \in\{1, \ldots, n\}: b_{i}=0, a_{i}=1\right\}\right|, \\
& m_{1}=\left|\left\{i \in\{1, \ldots, n\}: b_{i}=1, a_{i}=1\right\}\right| .
\end{aligned}
$$

Число векторов $a \in \mathbb{F}_{2}^{n}$ с фиксированными значениями $m_{0}$ и $m_{1}$ равно, очевидно, $C_{n-\mathrm{wt}(b)}^{m_{0}} C_{\mathrm{wt}(b)}^{m_{1}}$. Далее,

$$
\mathrm{d}(a, 0)=m_{0}+m_{1} \quad \text { и } \quad \mathrm{d}(a, b)=m_{0}+\mathrm{wt}(b)-m_{1},
$$

т. е. при $\operatorname{wt}(b) / 2 \leqslant r<n$ условия $\{\mathrm{d}(a, 0) \leqslant r, \mathrm{~d}(a, b) \leqslant r\}$ эквивалентны условиям

$$
\left\{\begin{array} { l } 
{ 0 \leqslant m _ { 0 } \leqslant n - \operatorname { w t } ( b ) , } \\
{ 0 \leqslant m _ { 1 } \leqslant \operatorname { w t } ( b ) , } \\
{ m _ { 0 } + m _ { 1 } \leqslant r , } \\
{ m _ { 0 } + \mathrm { wt } ( b ) - m _ { 1 } \leqslant r , }
\end{array} \Leftrightarrow \left\{\begin{array}{l}
0 \leqslant m_{0} \leqslant r-\operatorname{wt}(b) / 2, \\
0 \leqslant m_{1} \leqslant \operatorname{wt}(b), \\
\operatorname{wt}(b)-\left(r-m_{0}\right) \leqslant m_{1} \leqslant r-m_{0} .
\end{array}\right.\right.
$$

Значит,

$$
\begin{gathered}
N_{n}^{(2)}(\mathrm{wt}(\mathrm{b}), r)=\left|\left\{a \in \mathbb{F}_{2}^{n}: \max \{\mathrm{d}(a, 0), \mathrm{d}(a, b)\} \leqslant r\right\}\right|= \\
=\sum_{m_{0}=0}^{r-\mathrm{wt}(b) / 2} \sum_{m_{1}=\max \left\{\mathrm{wt}(b)-\left(r-m_{0}\right), 0\right\}}^{\min \left\{r-m_{0}, \mathrm{wt}(b)\right\}} C_{n-\mathrm{wt}(b)}^{m_{0}} C_{\mathrm{wt}(b)}^{m_{1}} .
\end{gathered}
$$

Чтобы получить верхнюю оценку для $N_{n}^{(2)}(i, r)$, заменим все слагаемые во внутренней сумме (4) их максимальным значением $C_{i}^{[i / 2]}$, положим $q_{i}=$ $=\frac{C_{n-i}^{r-[i / 2]-1}}{C_{n-i}^{r-[i / 2]}}=\frac{r-[i / 2]}{n-i+[i / 2]-r+1}$ и используем оценки $C_{n-i}^{r-[i / 2]-m} \leqslant q_{i}^{m} C_{n-i}^{r-[i / 2]}$. 
Если $i$ четное, то

$$
\begin{gathered}
N_{n}^{(2)}(i, r)=\sum_{m_{0}=0}^{r-i / 2} \sum_{m_{1}=\max \left\{i-\left(r-m_{0}\right), 0\right\}}^{\min \left\{r-m_{0}, i\right\}} C_{n-i}^{m_{0}} C_{i}^{m_{1}}< \\
<C_{i}^{i / 2} \sum_{m=0}^{r-i / 2} C_{n-i}^{r-i / 2-m}(1+2 m)<C_{i}^{i / 2} C_{n-i}^{r-i / 2} \sum_{m=0}^{\infty} q_{i}^{m}(1+2 m)= \\
=C_{i}^{i / 2} C_{n-i}^{r-i / 2} \frac{1+q_{i}}{\left(1-q_{i}\right)^{2}} .
\end{gathered}
$$

Если $i$ нечетное, то

$$
\begin{gathered}
N_{n}^{(2)}(i, r)=\sum_{m_{0}=0}^{r-[i / 2]} \sum_{m_{1}=\max \left\{i-\left(r-m_{0}\right), 0\right\}}^{\min \left\{r-m_{0}, i\right\}} C_{n-i}^{m_{0}} C_{i}^{m_{1}}< \\
<2 \cdot C_{i}^{[i / 2]} \sum_{m=0}^{r-[i / 2]} C_{n-i}^{r-[i / 2]-m}(m+1)< \\
<2 \cdot C_{i}^{[i / 2]} C_{n-i}^{r-[i / 2]} \sum_{m=0}^{\infty} q_{i}^{m}(m+1)=\frac{2 \cdot C_{i}^{[i / 2]} C_{n-i}^{r-[i / 2]}}{\left(1-q_{i}\right)^{2}} .
\end{gathered}
$$

Неравенство $q_{i}<q$ следует из того, что

$$
\begin{gathered}
q_{i}=\frac{r-[i / 2]}{n-i+[i / 2]-r+1}=1-\frac{n-2 r+1}{n-i+[i / 2]-r+1} \leqslant \\
\leqslant 1-\frac{n-2 r+1}{n-r+1}=\frac{r}{n-r+1}=q .
\end{gathered}
$$

Утверждение 2 доказано.

\section{§3. Коды с известными весовыми спектрами}

\section{Коды Рида-Мюллера}

Подпространство пространства $\mathbb{F}_{2}^{2^{m}}$, образованное векторами значений многочленов Жегалкина от $m$ переменных степени не выше $s$, называется двоичным кодом Рида-Мюллера длины $N=2^{m}$ и порядка $s$; для таких кодов используется обозначение $\mathrm{RM}(s, m)$.

В частности, кодовые слова кода Рида-Мюллера первого порядка $\mathrm{RM}(1, m)$ можно отождествить с табличными заданиями всех аффинных булевых функций от $m$ булевых переменных. Так как расстояния Хемминга 
между любыми двумя аффинными булевыми функциями от $m$ переменных равны либо $2^{m-1}$, либо $2^{m}$, то для кода $\mathrm{RM}(1, m)$ величины $\left|W_{i}\right|$ отличны от 0 только в трех случаях: $\left|W_{0}\right|=\left|W_{2^{m}}\right|=1,\left|W_{2^{m-1}}\right|=2^{m+1}-2$. Приведенная в следующей теореме оценка для $r$-окрестности кода $\mathrm{RM}(1, m)$ в терминах окрестностей булевых функций доказана в [39] и является следствием теоремы 1 настоящей работы.

Теорема 3. Если $m \geqslant 2$, то

$$
\left|\mathbb{F}_{2}(R M(1, m), r)\right|=(1-Q(m, r)) 2^{m+1} \sum_{i=0}^{r} C_{2^{m}}^{i},
$$

где

$$
\begin{gathered}
0 \leqslant Q(m, r)<\frac{2^{m} N_{2^{m}}^{(2)}\left(2^{m-1}, r\right)}{\sum_{i=0}^{r} C_{2^{m}}^{i}} \leqslant \\
\leqslant \frac{2^{m} C_{2^{m-1}}^{2^{m-2}} C_{2^{m-1}}^{r-2^{m-2}}}{C_{2^{m}-1}^{r-1}} \frac{\left(2^{m-1}+1\right)\left(3 \cdot 2^{m-2}-r+1\right)}{\left(2^{m}-2 r+1\right)^{2}}
\end{gathered}
$$

в частности, $Q(m, r)=0$ при $0 \leqslant r<2^{m-2} u$

$$
0<Q(m, r)<\frac{1}{15} 2^{-\left(c_{r}^{2}-\frac{3}{2}\right) m} \exp \left\{\frac{2\left(c_{r}^{2} m\right)^{3 / 2}}{2^{m / 2}}\right\}
$$

nрu $m \geqslant 8, r=2^{m-1}-c_{r} \sqrt{2^{m-1} m \ln 2} \geqslant 0, c_{r}>1$.

Аналогичная теорема справедлива для кода Рида-Мюллера второго порядка $\mathrm{RM}(2, m)$. Спектр расстояний этого кода имеет более сложный вид (см., например, [42], § 15.2, с. 426):

$$
\left|W_{i}\right|= \begin{cases}1, & \text { если } i=0 \text { или } i=2^{m}, \\ \frac{2^{s(s+1)}\left(2^{m}-1\right)\left(2^{m-1}-1\right) \ldots\left(2^{m-2 s+1}-1\right)}{\left(2^{2 s}-1\right)\left(2^{2 s-2}-1\right) \ldots\left(2^{2}-1\right)}, & \left|i-2^{m-1}\right|=2^{m-s-1}, 1 \leqslant s \leqslant \frac{m}{2}, \\ 2^{C_{m}^{2}+m+1}-\sum_{i \neq 2^{m-1}}\left|W_{i}\right|, & \text { если } i=2^{m-1}, \\ 0, & \text { в остальных случаях. }\end{cases}
$$

Следующая теорема (в терминах окрестностей множества булевых функций степени не выше 2) доказана в [43]; см. также [44]. Ввиду громоздкости формул оценки величины $Q(m, r)$ в терминах комбинаторных сумм (как в теореме 3) не приводятся. 
Теорема 4. Если $m \geqslant 3$, то

$$
\left|\mathbb{F}_{2}(\mathrm{RM}(2, m), r)\right|=(1-Q(m, r)) 2^{\left(\begin{array}{c}
m \\
2
\end{array}\right)+m+1} \sum_{i=0}^{r} C_{2^{m}}^{i},
$$

где $Q(m, r)=0$ при $0 \leqslant r<2^{m-3}$,

$$
0<Q(m, r)<\frac{2^{-m^{2}\left(c_{r}^{2}-3\right) / 6+m+1}}{m^{2}} \exp \left\{\frac{\left(c_{r} m\right)^{3}}{7 \cdot 2^{m / 2}}\right\}
$$

nрu $m \geqslant 15$ u $r=2^{m-1}-c_{r} m \sqrt{2^{m-2} \ln 2} \geqslant 0, c_{r}>1$.

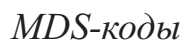

Пусть $\mathcal{C}-[n, k, d]$-код над $G F(q)$ с проверочной матрицей $\mathbf{H}$ и порождающей матрицей $\mathbf{G}$. Для полноты изложения сформулируем и докажем теорему 5 .

Теорема 5 (граница Синглтона; [42, с. 43]). Если $\mathcal{C}-[n, k, d]$-код, то

$$
n-k+1 \geqslant d \text {. }
$$

Доказательство. Ранг проверочной матрицы $\mathbf{H}$ кода $\mathcal{C}$, т. е. максимальное число ее линейно независимых столбцов, равен $n-k$. Значит, любые $n-k+1$ столбцов являются линейно зависимыми. Поэтому в $\mathcal{C}$ существует ненулевой вектор, все координаты которого, не соответствующие $n-k+1$ линейно зависимым столбцам, равны 0. Вес Хемминга такого вектора не больше $n-k+1$, что и требовалось доказать.

Определение. Коды, для которых достигается граница Синглтона $d=$ $n-k+1$, называются MDS-кодами (maximum distance separable codes) или МДР-кодами, т. е. максимальными дистаниионно разделимыми кодами).

Известно много конструкций MDS-кодов. В частности, $q$-значные коды Рида-Соломона (коды Боуза-Чоудхури-Хоквингема над $\mathrm{GF}(q)$, см. ниже) являются MDS-кодами.

Перечислим основные свойства MDS-кодов.

Теорема 6 ([42, с. 310]). Код $\mathcal{C}$ является MDS-кодом тогда и только тогда, когда любые $n-k$ столбиов матриць $\mathbf{H}$ линейно независимы.

Теорема 7 ([42, с. 311]). Если $\mathcal{C}-M D S$-код, то дуальный ему код $\mathcal{C}^{\perp}$ также является MDS-кодом.

Доказательство. Пусть $\mathcal{C}-$ MDS-код размерности $k$ и длины $n$. Его проверочная матрица Н размера $(n-k) \times n$ имеет ранг $n-k$ и является порождающей матрицей дуального кода $\mathcal{C}^{\perp}$ размерности $n-k$.

2013. T. 4. № 2. C. 17-42 
Согласно теореме 6, любые $n-k$ столбцов матрицы $\mathbf{H}$ образуют невырожденную квадратную матрицу. Поэтому линейная комбинация строк матрицы Н может иметь $n-k$ нулевых координат, только если все ее коэффициенты равны 0. Значит, в любых $n-k$ позициях кодовых слов из $\mathcal{C}^{\perp}$ содержится хотя бы один ненулевой элемент. Следовательно, каждое кодовое слово кода $\mathcal{C}^{\perp}$ имеет не меньше $k+1$ ненулевых координат. Но в силу границы Синглтона кодовое расстояние кода $\mathcal{C}^{\perp}$ удовлетворяет неравенству

$$
d \leqslant n-(n-k)+1=k+1,
$$

значит, минимальное расстояние кода $\mathcal{C}^{\perp}$ равно $k+1$.

Следствие 1. Для кода С следующие утверждения эквивалентны:

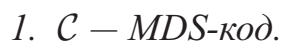

2. Любые k столбиов порождающей матрищь $\mathbf{G}$ линейно независимы.

3. Любые $n-k$ столбиов проверочной матрищы $\mathbf{H}$ линейно независимы.

Двоичный MDS-код может иметь только параметры вида $[n, 1, n]$, $[n, n-1,2]$ или $[n, n, 1]$. Такие коды называются тривиальными MDS-кодами.

Код с параметрами $[n, k, d]$ называется нетривиальным, если $2 \leqslant k \leqslant n-2$.

Описание спектров весов Хемминга MDS-кодов, а значит, и их спектров расстояний, содержится в следующей теореме.

Теорема 8 ([42, с.312]). Число кодовых слов веса $\omega$ в MDS-коде $\mathcal{C}$ с параметрами $[n, k, d=n-k+1]$ над полем $G F(q)$ определяется равенствами

$$
\begin{gathered}
A_{w}=\left(\begin{array}{c}
n \\
w
\end{array}\right) \sum_{j=0}^{w-d}(-1)^{j}\left(\begin{array}{c}
w \\
j
\end{array}\right)\left(q^{w-d+1-j}-1\right)= \\
=\left(\begin{array}{c}
n \\
w
\end{array}\right)(q-1) \sum_{j=0}^{w-d}(-1)^{j}\left(\begin{array}{c}
w-j \\
j
\end{array}\right) q^{w-d-j} .
\end{gathered}
$$

Пользуясь тем, что число $A_{n-k+2}=\left(\begin{array}{c}n \\ k-2\end{array}\right)(q-1)(q-n+k-1)$ неотрицательно, нетрудно убедиться в справедливости следующего утверждения.

Следствие 2 ([42, с. 312]). Пусть MDS-код $\mathcal{C}$ имеет параметры $[n, k, n-k+1]$. Если $k \geqslant 2$, то $q \geqslant n-k+1$. Если $k \leqslant n-2$, то $q \geqslant k+1$. 
Теорема 9 ([42, с. 311]). Если $\mathcal{C}-[n, k, d]$-код над $G F(q)$, то $\mathcal{C}$ является MDS-кодом тогда и только тогда, когда для любых $d$ позиций в коде $\mathcal{C}$ найдется слово минимального веса, ненулевые элементы которого занимают эти позиции; в этом случае $d=n-k+1$.

Следствие 3. Число кодовых слов веса $n-k+1$ в коде $\mathcal{C}$ равно

$$
(q-1)\left(\begin{array}{c}
n \\
n-k+1
\end{array}\right) \text {. }
$$

Beса кодовых слов в MDS-коде с параметрами $[n, k, d]$ принимают не более $k$ различных значений от $n-k+1$ до $n$.

Построение MDS-кодов связано с комбинаторными задачами, которые во многих случаях еще не решены. Ряд примеров MDS-кодов содержится в [42]. Приведем здесь только 2 примера. Если $\alpha_{1}, \ldots, \alpha_{q-1}-$ ненулевые элементы поля $\mathrm{GF}(q)$, то

$$
\left(\begin{array}{cccc}
1 & \ldots & 1 & 1 \\
\alpha_{1} & \ldots & \alpha_{q-1} & 0 \\
\alpha_{1}^{2} & \ldots & \alpha_{q-1}^{2} & 0 \\
\ldots & \ldots & \ldots & \ldots \\
\alpha_{1}^{q-k+1} & \ldots & \alpha_{q-1}^{q-k+1} & 0
\end{array}\right), \quad\left(\begin{array}{ccccc}
1 & \ldots & 1 & 1 & 0 \\
\alpha_{1} & \ldots & \alpha_{q-1} & 0 & 0 \\
\alpha_{1}^{2} & \ldots & \alpha_{q-1}^{2} & 0 & 0 \\
\ldots & \ldots & \ldots & \ldots & \ldots \\
\alpha_{1}^{q-k+1} & \ldots & \alpha_{q-1}^{q-k+1} & 0 & 1
\end{array}\right)
$$

являются проверочными матрицами MDS-кодов с параметрами $[q, k, q-k+1]$ и $[q+1, k, q-k+2]$.

\section{Циклические и БЧХ коды}

Коды БЧХ (Боуза-Чоудхури-Хоквингема) являются частными случаями линейных изиклических кодов, т. е. кодов, которые вместе с кодовым словом $\left(c_{n-1}, c_{n-2}, \ldots, c_{1}, c_{0}\right)$ содержат все его циклические сдвиги

$$
\left(c_{0}, c_{n-1}, c_{n-2}, \ldots, c_{1}\right),\left(c_{1}, c_{0}, c_{n-1}, c_{n-2}, \ldots, c_{2}\right), \ldots,\left(c_{n-2}, \ldots, c_{0}, c_{n-1}\right) .
$$

Кодовые слова циклического кода длины $n$, в котором координаты кодовых слов рассматриваются как элементы конечного поля $\mathbb{F}$, удобно рассматривать как набор коэффициентов многочлена из кольца $R_{n}=\mathbb{F}[x] /\left(x^{n}-1\right)$ :

$$
\left(c_{n-1}, c_{n-2}, \ldots, c_{1}, c_{0}\right) \leftrightarrow c_{0}+c_{1} x+\ldots+c_{n-1} x^{n-1} .
$$

В этом кольце умножение на $x$ соответствует циклическому сдвигу коэффициентов многочлена:

$$
x\left(c_{0}+c_{1} x+\ldots+c_{n-1} x^{n-1}\right)=c_{n-1}+c_{0} x+\ldots+c_{n-2} x^{n-1} \quad\left(\bmod x^{n}-1\right) .
$$


При такой интерпретации циклический код можно определить как идеал кольца $R_{n}$, т.е. как линейное подпространство кольца $R_{n}$, замкнутое относительно умножения на многочлены $r(x) \in R_{n}$.

Каждый идеал $\mathcal{C}$ кольца $R_{n}$ является главным, т. е. все его элементы являются кратными некоторого порождающего многочлена $g(x) \in R_{n}$. Порождающий многочлен $g(x)$ имеет минимальную степень среди ненулевых элементов $\mathcal{C}$, является делителем многочлена $x^{n}-1$, и коэффициент при старшем члене $g(x)$ равен 1 (см. [42, § 7.3, с. 191]). Циклический код $\mathcal{C}$ как линейный код в пространстве $\mathbb{F}^{n}$ порождается матрицей, строки которой соответствуют многочленам

$$
g(x), x g(x) \ldots, x^{n-r-1} g(x) .
$$

Строки проверочной матрицы такого кода соответствуют произведениям $x^{m} h(x)$, где $x^{n-\operatorname{deg} g(x)} h\left(x^{-1}\right)=\left(x^{n}-1\right) / g(x)$.

Каждый код БЧХ длины $n$ над $\mathrm{GF}(q)$ порождается многочленом $g(x)$, который удовлетворяет следующему условию: если $\alpha-$ примитивный корень многочлена $x^{n}-1$, лежащий в некотором расширении $\operatorname{GF}\left(q^{m}\right)$ поля $\operatorname{GF}(q)$, то существуют такие целые числа $b \geqslant 0, \delta \geqslant 2$, что $\alpha^{b}, \alpha^{b+1}, \ldots, \alpha^{b+\delta-2}$ являются корнями $g(x)$. В этом случае минимальное расстояние БЧХ-кода не меньше конструктивного кодового расстояния $\delta$.

В [45] получено асимптотическое представление некоторых элементов весового спектра БЧХ-кодов, а значит, и спектра расстояний, так как БЧХкод - линейный.

Теорема 10 ([45, с. 221]). Пусть $p>2, \mathcal{C}=B C H\left(B_{\mathbb{F}_{q}}^{(d)}\right)-p$-значный БЧХ-код длины $q=p^{l}$ с конструктивным кодовым расстоянием $d$, определяемый проверочной матрицей $B_{\mathbb{F}_{q}}^{(d)}$ и пусть $r_{d}=1+l \cdot\left(d-2-\left[\frac{d-2}{p}\right]\right)-$ размерность дуального кода $B C H^{\perp}\left(B_{\mathbb{F}_{q}}^{(d)}\right)$. Тогда при

$$
d=\text { const }, \quad q \rightarrow \infty \quad u \quad i>2\left(d-2-\left[\frac{d-2}{p}\right]\right)
$$

имеет место следующее асимптотическое представление элемента $A_{i}$ спектра кода ВСH(B $\left.B_{\mathbb{F}_{q}}^{(d)}\right)$ :

$$
A_{i}=p^{-r_{d}}\left((p-i)^{i}+(-1)^{i}(p-1)\right)\left(\begin{array}{c}
q \\
i
\end{array}\right)\left(1+\varepsilon_{i, q}\right),
$$

где $\varepsilon_{i, q} \asymp q^{-1 / 4} p^{r_{d}}(p-1)^{-i}\left(\begin{array}{l}q \\ i\end{array}\right)^{-1 / 2} \rightarrow 0$ при $q \rightarrow \infty$.

В [46] получены асимптотические формулы для числа кодовых слов определенного веса в БЧХ-коде $\mathcal{C}$ длины $n=2^{l}-1$ с конструктивным кодовым расстоянием $2 t+1$. 
При $2 t+\nu(t) \leqslant i \leqslant n-(2 t+\nu(t))$, где

$$
\nu(t)=] \frac{2 t \ln t+4 \frac{1}{2} t+0.1 \ln n}{\frac{1}{2} \ln n-\ln t-\frac{9}{4}}[\text {, }
$$

и $0<t<0.1 \sqrt{n}$, показано (см. [46]), что $A_{i}=(n+1)^{-t}\left(\begin{array}{c}n \\ i\end{array}\right)(1+\varepsilon),|\varepsilon|<$ const $\times$ $\times n^{-0.1}$. Заметим, что при $t \leqslant 0.2 \frac{\ln n}{\ln \ln n}$ и достаточно больших $n$ функция $\nu(t)$ равна 1.

Статья [5] содержит ряд известных и новых результатов для весовых энумераторов неприводимых циклических кодов. В формулировках этих результатов циклические коды задаются способом, отличающимся от использованного в начале этого пункта

Пусть $p$ - простое число, $q=p^{s}, N \in \mathbb{N}, N>1, r=q^{m}, N \mid(r-1)$ и $n=(r-1) / N$. Пусть $\alpha$ - примитивный элемент $\mathrm{GF}(r)$ и $\theta=\alpha^{N}$. Множество

$$
\mathcal{C}(r, N)=\left\{\left(\operatorname{Tr}_{r / q}(\beta), \operatorname{Tr}_{r / q}(\beta \theta), \ldots, \operatorname{Tr}_{r / q}\left(\beta \theta^{n-1}\right)\right) \mid \beta \in \mathrm{GF}(r)\right\}
$$

называется неприводимым цүиклическим $\left[n, m_{0}\right]$ кодом над $\mathrm{GF}(q)$, где $m_{0}-$ мультипликативный порядок $q$ по модулю $n, m_{0}$ делит $m, \operatorname{Tr}_{r / q}: \mathrm{GF}(r) \rightarrow$ $\rightarrow \mathrm{GF}(q)$ - функция след, переводящая каждый элемент $\beta \in \mathrm{GF}(r)$ в элемент $\beta+\beta^{q}+\ldots+\beta^{q^{m-1}} \in \mathrm{GF}(q)$.

Теорема 11 ([5]). а) Если НОД $((r-1) /(q-1), N)=1$, то множество $C(r, N)$ является $\left[\left(q^{m}-1\right) / N, m,(q-1) q^{m-1} / N\right]$-кодом постоянного веса с весовым энумератором

$$
1+(r-1) x^{\frac{(q-1) q^{m-1}}{N}}
$$

б) Если НОД $((r-1) /(q-1), N)=2$, то $C(r, N)-$ двухвесовой $\left[\left(q^{m}-\right.\right.$ $-1) / N, m,(q-1)(r-\sqrt{r}) / N q]$-код с весовым энумератором

$$
1+\frac{r-1}{2} x^{\frac{(q-1)(r-\sqrt{r})}{N q}}+\frac{r-1}{2} x^{\frac{(q-1)(r+\sqrt{r})}{N q}} .
$$

в) Если НОД $((r-1) /(q-1), N)=3$, то $C(r, N)-\left[\left(q^{m}-1\right) / N, m\right]-\kappa о д$ с весовым энумератором

$$
1+\frac{r-1}{3} x^{\frac{(q-1)\left(r-c_{1} \sqrt[3]{r}\right)}{N q}}+\frac{r-1}{3} x^{\frac{(q-1)\left(r+\frac{1}{2}\left(c_{1}+9 d_{1}\right) \sqrt[3]{r}\right)}{N q}}+\frac{r-1}{3} x^{\frac{(q-1)\left(r+\frac{1}{2}\left(c_{1}-9 d_{1}\right) \sqrt[3]{r}\right)}{N q}} .
$$

2013. T. 4. № 2. C. 17-42 
г) Если НОД $((r-1) /(q-1), N)=4 u p \equiv 1(\bmod 4)$, то $C(r, N)-$ $[(r-1) / N, m]$-код с весовым энумератором

$$
\begin{aligned}
1 & +\frac{r-1}{4} x^{\frac{(q-1)\left(r+\sqrt{r}+2 u_{1} \sqrt[4]{r}\right)}{N q}}+\frac{r-1}{4} x^{\frac{(q-1)\left(r+\sqrt{r}-2 u_{1} \sqrt[4]{r}\right)}{N q}}+ \\
& +\frac{r-1}{4} x^{\frac{(q-1)\left(r-\sqrt{r}+4 v_{1}\right.}{N q}}+\frac{\sqrt[4]{r})}{4} x^{\left.\frac{r-1)\left(r-\sqrt{r}-4 v_{1}\right.}{N q} \sqrt[4]{r}\right)}
\end{aligned}
$$

где $u_{1}$ и $v_{1}$ удовлетворяют условиям $q^{m / 2}=u_{1}^{2}+4 v_{1}^{2}, u_{1} \equiv 1(\bmod 4)$, $u$ НОД $\left(u_{1}, p\right)=1$.

д) Если НОД $((r-1) /(q-1), N)=4 u p \equiv 3(\bmod 4), \operatorname{mo} C(r, N)-$ двухвесовой $[(r-1) / N, m]$-код с весовым энумератором

$$
1+3 \frac{r-1}{4} x^{\frac{(q-1)(r-\sqrt{r})}{N q}}+\frac{r-1}{4} x^{\frac{(q-1)(r+3 \sqrt{r})}{N q}} .
$$

ЗАМЕЧАНИЕ 1. Существование корней из $r$ следует из условий на НОД чисел $(r-1) /(q-1)$ и $N$.

В [31] описан весовой энумератор неприводимого циклического кода длины $n$, где $n$ - степень простого числа.

Теорема 12. Пусть $t$ - простое, $l$ - положительное целое и мультипликативный порядок $q$ по модулю $t^{l}$ равен $t^{d}, 0 \leqslant d<l$. Положим $m=t^{d}$ u $N=\left(q^{m}-1\right) / t^{j}$ для любого $1 \leqslant j<l$.

Если $j \leqslant l-d$, то $C(r, N)-\left[t^{j}, 1, t^{j}\right]$ код постоянного веса над $\mathrm{GF}(q)$ с весовым энумератором

$$
1+(q-1) x^{t^{j}}
$$

Если $j>l-d$, то $C(r, N)-\left[t^{j}, t^{j-(l-d)}\right]$ циклический код над $\operatorname{GF}(q)$ с весовым энумератором

$$
\sum_{\omega=0}^{t^{r-l+d}}\left(\begin{array}{c}
t^{j-l+d} \\
\omega
\end{array}\right) x^{t^{(l-d) \omega}}
$$

Теорема 13 описывает класс неприводимых циклических кодов над $\mathrm{GF}(q), q=p^{s}, p-$ простое, содержащих слова двух весов.

Теорема 13 ([5]). Пусть sm четно, $N>0, N \mid(r-1) u N_{1}=$ НОД $((r-$ $-1) /(q-1), N)>2$. Пусть $j>0-$ минимальное число для которого $p^{j} \equiv$ $\equiv-1\left(\bmod N_{1}\right), u \gamma=s m / 2 j$. 
а) Если $\gamma, p$ и $\left(p^{j}+1\right) / N_{1}$ нечетные, то $C(r, N)$ - это

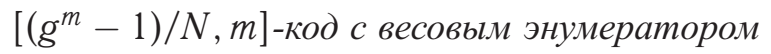

$$
1+\frac{r-1}{N_{1}} x^{\frac{(q-1)\left(r-\left(N_{1}-1\right) \sqrt{r}\right)}{N q}}+\frac{(r-1)\left(N_{1}-1\right)}{N_{1}} x^{\frac{(q-1)(r+\sqrt{r})}{N q}}
$$

при условии $N_{1}<\sqrt{r}+1$.

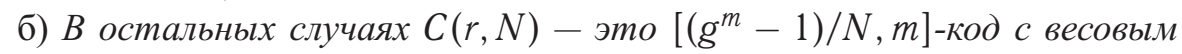
энумератором

$$
1+\frac{r-1}{N_{1}} x^{\frac{(q-1)\left(r+(-1)^{\gamma}\left(N_{1}-1\right) \sqrt{r}\right)}{N q}}+\frac{(r-1)\left(N_{1}-1\right)}{N_{1}} x^{\frac{(q-1)\left(r-(-1)^{\gamma}\left(N_{1}-1\right) \sqrt{r}\right)}{N q}}
$$

при условии $\sqrt{r}+(-1)^{\gamma}\left(N_{1}-1\right)>0$.

Теорема 14 содержит явные оценки весов кодовых слов в неприводимом циклическом коде; такие оценки дают информацию о возможности исправления ошибок кодами из этого класса.

Теорема 14 ([5]). Пусть $N \mid(r-1), N_{1}=\operatorname{HОД}((r-1) /(q-1), N)$ и мультипликативный порядок q по модулю $n$ равен $m_{0}$. Тогда $C(r, N)-$ ичикический код с параметрами $\left[\left(g^{m}-1\right) / N, m_{0}\right]$ над $\mathrm{GF}(q)$, и для любого $c \in C(r, N) \backslash\{0\}$

$$
\begin{aligned}
& \omega(c) \geqslant(q-1)\left\lceil\frac{r-\left\lfloor\left(N_{1}-1\right) \sqrt{r}\right\rfloor}{N q}\right\rfloor, \\
& \omega(c) \leqslant(q-1)\left\lfloor\frac{\left.r+\left\lfloor\left(N_{1}-1\right) \sqrt{r}\right)\right\rfloor}{N q}\right\rfloor,
\end{aligned}
$$

где $\omega(\cdot)$ - весовая функиия Хемминга. В частности, если $N_{1}\left(N_{1}-1\right)<r$, то $m_{0}=m$.

\section{Коды постоянного веса}

В [4] для любого нечетного $q=p^{n}, p-$ простое, построен нелинейный двоичный код $C(q, 2)$ длины $q$, в котором все $\frac{q^{2}-q}{2}$ кодовых слов имеют вес $\frac{q-1}{2}$. Расстояние Хемминга между любыми словами из $C(q, 2)$ лежит в интервале $\left[\frac{q}{2}-\frac{3}{2} \sqrt{q}, \frac{q}{2}+\frac{3}{2} \sqrt{q}\right]$. Показано также, что код $C(q, 2)$ является дистанционно-инвариантным.

В [34] приведены таблицы кодов длины $29 \leqslant n \leqslant 63$ со словами фиксированного веса $5 \leqslant \omega \leqslant 8$ с минимальным расстоянием $d=2 \omega-2, d=2 \omega-4$ и $d=2 \omega-6$. Такие таблицы уже были построены для $n \leqslant 28$. Ограничение длины величиной 63 было выбрано, поскольку это максимально возможное количество частот в мобильных телефонных системах GSM, когда используется перескок частоты.

2013. T. 4. № 2. C. 17-42 
В работе [23] рассматривается задача поиска бинарных кодов длины $n$ постоянного веса $\omega$ и минимальным расстоянием $d$ с максимально возможным количеством кодовых слов $A(n, d, \omega)$. Подробное описание верхних оценок $A(n, d, \omega)$ можно найти в [1]; нижние оценки $A(n, d, \omega)$ обычно являются конструктивными. В [23] найдены коды, улучшающие нижние оценки $A(n, d, \omega)$, длины $29 \leqslant n \leqslant 63$. В частности, получены 10 новых оптимальных кодов $(d=n-k+1)$, которые не были известны ранее.

\section{Kодьн над $\mathbb{Z}_{4}$}

В [9] содержится краткое введение в коды над $\mathbb{Z}_{4}$, описание линейных кодов Кердока и Препараты над $\mathbb{Z}_{4}$, а также некоторых других связанных с ними кодов. Материал этой статьи используется в дальнейшем изложении.

Коды Кердока и Препараты дистанционно-инвариантны, поэтому их весовые спектры совпадают со спектрами расстояний.

В [42, с. 440], для четного $m, m \geqslant 4$, приведен спектр весов кода Кердока $\mathcal{C}(m)$ (нелинейного кода над $\mathbb{Z}_{2}$ длины $n=2^{m}$, мощности $2^{2 m}$, с минимальным расстоянием $\left.2^{m-1}-2^{(m-2) / 2}\right)$ :

\begin{tabular}{|c|c|c|c|c|c|}
\hline$i$ & 0 & $2^{m-1}-2^{(m-2) / 2}$ & $2^{m-1}$ & $2^{m-1}+2^{(m-2) / 2}$ & $2^{m}$ \\
\hline$A_{i}$ & 1 & $2^{m}\left(2^{m-1}-1\right)$ & $2^{m+1}-2$ & $2^{m}\left(2^{m-1}-1\right)$ & 1 \\
\hline
\end{tabular}

Код Кердока является объединением кода $R(1, m)$ и $2^{m-1}-1$ смежных классов кода $R(2, m)$ по $R(1, m)$. Кроме того, $R(1, m) \subset \mathcal{C}(m) \subset R(2, m)$.

Интересен следующий факт: для некоторых $n$ и $d$ можно строить нелинейные коды с бо́льшим количеством кодовых слов, чем это возможно для линейных кодов. Например, для $n=16$ и $d=6$ линейный код с максимальным количеством кодовых слов имеет размерность $k=7$, т. е. 128 кодовых слов. В 1967 году Нордстром и Робинсон нашли нелинейный код с параметрами $n=16$ и $d=6$, содержащий 256 кодовых слов.

Препарата [28] обобщил эту конструкцию на бесконечное семейство кодов с параметрами $\left(2^{m+1}, 2^{2^{m+1}-2 m-2}, 6\right), m-$ нечетное, $m \geqslant 3$. Несколькими годами позже, в 1972 году, Кердок [13] дал другое обобщение кода Нордстрома-Робинсона и построил другой бесконечный класс кодов с параметрами $\left(2^{m+1}, 2^{2 m+2}, 2^{m}-2^{\frac{m-1}{2}}\right), m-$ нечетное, $m \geqslant 3$.

В [8] было получено простое описание семейства кодов Кердока с помощью отображения Грея $\psi: \mathbb{Z}_{4} \rightarrow \mathbb{Z}_{2}^{2}$, определяемого следующим образом:

$$
\psi(0)=00, \quad \psi(1)=01, \quad \psi(2)=11, \quad \psi(3)=10 .
$$


Для элементов $\mathbb{Z}_{4}$ вводится вес Ли:

$$
\omega_{L}(0)=0, \quad \omega_{L}(1)=1, \quad \omega_{L}(2)=2, \quad \omega_{L}(3)=1 .
$$

При таком определении естественное (покоординатное) продолжение отображения $\psi$ до отображения $\psi^{n}: \mathbb{Z}_{4}^{n} \rightarrow \mathbb{Z}_{2}^{2 n}$ оказывается изометричным (сохраняющим расстояния) отображением из $\mathbb{Z}_{4}^{n}$ (с метрикой Ли) в $\mathbb{Z}_{2}^{2 n}$ (с метрикой Хемминга). Поэтому отображение $\psi^{n}$ переводит любой линейный код $\mathcal{C}$ длины $n$ над $\mathbb{Z}_{4}$ в бинарный нелинейный код $\mathcal{C}=\psi^{n}(\mathcal{C})$ длины $2 n$ с сохранением попарных расстояний между кодовыми словами, и весовые спектры этих кодов совпадают.

Итак, пусть $\mathcal{C}-$ линейный код над $\mathbb{Z}_{4}$; тогда существует дуальный к нему код $\mathcal{C}^{\perp}$. Из изометричности отображения $\psi^{n}$ следует, что тождество Мак-Вильямс справедливо для нелинейных кодов $\mathcal{C}_{*}=\psi^{n}(\mathcal{C})$ и $\mathcal{C}_{*}^{\perp}=\psi^{n}\left(\mathcal{C}^{\perp}\right)$ над $\mathbb{Z}_{2}$.

Код Нордстрома-Робинсона $\mathcal{N}_{16}$ с параметрами $[16,256,6]$, который эквивалентен коду Кердока при $m=4(\mathcal{C}(4))$, является дистанционноинвариантным и имеет весовой спектр

\begin{tabular}{|c|c|c|c|c|c|}
\hline$i$ & 0 & 6 & 8 & 10 & 16 \\
\hline$A_{i}$ & 1 & 112 & 30 & 112 & 1 \\
\hline
\end{tabular}

В [8] показано, что такой бинарный код может быть построен из линейного кода над $\mathbb{Z}_{4}$ с помощью отображения Грея.

Пусть $\mathcal{C}$ - линейный код над $\mathbb{Z}_{4}$ и $d_{H}\left(d_{L}\right)$ - минимальное расстояние Хемминга (Ли) кода $\mathcal{C}$. В [29] показано, что $d_{H} \geqslant\left\lceil\frac{d_{L}}{2}\right\rceil$. Если $d_{H}=\left\lceil\frac{d_{L}}{2}\right\rceil$, то говорят, что $\mathcal{C}$ имеет тип $\alpha$, в противном случае - что $\mathcal{C}$ имеет тип $\beta$. В [7] построены линейные симплексные коды типов $\alpha$ и $\beta$ над $\mathbb{Z}_{4}$ : код $S_{k}^{\alpha}$ с параметрами $\left[2^{2 k}, 2 k, d_{H}=2^{2 k-1}, d_{L}=2^{2 k}\right]$ и код $S_{k}^{\beta}$ с параметрами $\left[2^{k-1}\left(2^{k}-1\right), 2 k, d_{H}=2^{2 k-2}, d_{L}=2^{k-1}\left(2^{k}-1\right)\right]$. Показано, что образы $\psi\left(S_{k}^{\alpha}\right)$ и $\psi\left(S_{k}^{\beta}\right)$ этих кодов при отображении Грея не являются линейными кодами над $\mathbb{Z}_{2}$.

Теорема 15. Код $S_{k}^{\alpha}$ имеет следующие спектры весов Хемминга (Ли):

1. $A_{0}^{H}=1, A_{2^{2 k-1}}^{H}=2^{k}-1, A_{3 \cdot 2^{2 k-2}}^{H}=2^{k}\left(2^{k}-1\right)$;

2. $A_{0}^{L}=1, A_{2^{2 k}}^{L}=2^{2 k}-1$.

Аналогичные теоремы имеют место для кодов $S_{k}^{\alpha}, S_{k}^{\beta}$ и кода $\mathrm{RM}(1, m-$ $s+1)$ над $\mathbb{Z}_{2^{s}}$. 


\section{Коды Голея}

Линейный двоичный расширенный код Голея $\mathcal{G}_{24}$ с параметрами $[24,12,8]$ порождается матрицей

$\left(\begin{array}{llllllllllllllllllllllll}1 & 1 & 0 & 0 & 0 & 0 & 0 & 0 & 0 & 0 & 0 & 0 & 0 & 1 & 1 & 0 & 1 & 1 & 1 & 0 & 0 & 0 & 1 & 0 \\ 1 & 0 & 1 & 0 & 0 & 0 & 0 & 0 & 0 & 0 & 0 & 0 & 0 & 0 & 1 & 1 & 0 & 1 & 1 & 1 & 0 & 0 & 0 & 1 \\ 1 & 0 & 0 & 1 & 0 & 0 & 0 & 0 & 0 & 0 & 0 & 0 & 0 & 1 & 0 & 1 & 1 & 0 & 1 & 1 & 1 & 0 & 0 & 0 \\ 1 & 0 & 0 & 0 & 1 & 0 & 0 & 0 & 0 & 0 & 0 & 0 & 0 & 0 & 1 & 0 & 1 & 1 & 0 & 1 & 1 & 1 & 0 & 0 \\ 1 & 0 & 0 & 0 & 0 & 1 & 0 & 0 & 0 & 0 & 0 & 0 & 0 & 0 & 0 & 1 & 0 & 1 & 1 & 0 & 1 & 1 & 1 & 0 \\ 1 & 0 & 0 & 0 & 0 & 0 & 1 & 0 & 0 & 0 & 0 & 0 & 0 & 0 & 0 & 0 & 1 & 0 & 1 & 1 & 0 & 1 & 1 & 1 \\ 1 & 0 & 0 & 0 & 0 & 0 & 0 & 1 & 0 & 0 & 0 & 0 & 0 & 1 & 0 & 0 & 0 & 1 & 0 & 1 & 1 & 0 & 1 & 1 \\ 1 & 0 & 0 & 0 & 0 & 0 & 0 & 0 & 1 & 0 & 0 & 0 & 0 & 1 & 1 & 0 & 0 & 0 & 1 & 0 & 1 & 1 & 0 & 1 \\ 1 & 0 & 0 & 0 & 0 & 0 & 0 & 0 & 0 & 1 & 0 & 0 & 0 & 1 & 1 & 1 & 0 & 0 & 0 & 1 & 0 & 1 & 1 & 0 \\ 1 & 0 & 0 & 0 & 0 & 0 & 0 & 0 & 0 & 0 & 1 & 0 & 0 & 0 & 1 & 1 & 1 & 0 & 0 & 0 & 1 & 0 & 1 & 1 \\ 1 & 0 & 0 & 0 & 0 & 0 & 0 & 0 & 0 & 0 & 0 & 1 & 0 & 1 & 0 & 1 & 1 & 1 & 0 & 0 & 0 & 1 & 0 & 1 \\ 0 & 0 & 0 & 0 & 0 & 0 & 0 & 0 & 0 & 0 & 0 & 0 & 1 & 1 & 1 & 1 & 1 & 1 & 1 & 1 & 1 & 1 & 1 & 1\end{array}\right)$

Код Голея $\mathcal{G}_{23}$ с параметрами $[23,12,7]$ получается выбрасыванием последней координаты из каждого слова кода $\mathcal{G}_{24}$.

Коды Голея обладают рядом уникальных свойств; многие из них описаны в [42]. Код $\mathcal{G}_{24}$ имеет весовой спектр

\begin{tabular}{|c|c|c|c|c|c|}
\hline$i$ & 0 & 8 & 12 & 16 & 24 \\
\hline$A_{i}$ & 1 & 759 & 2576 & 759 & 1 \\
\hline
\end{tabular}

Код $\mathcal{G}_{23}$ имеет весовой спектр

\begin{tabular}{|c|c|c|c|c|c|c|c|c|}
\hline$i$ & 0 & 7 & 8 & 11 & 12 & 15 & 16 & 23 \\
\hline$A_{i}$ & 1 & 253 & 506 & 1288 & 1288 & 506 & 253 & 1 \\
\hline
\end{tabular}

Код Голея $\mathcal{G}_{23}$ исправляет любые три ошибки и является совершенным: для любого слова $x \in \mathbb{Z}_{2}^{23}$ существует ровно одно кодовое слово из $\mathcal{G}_{23}$, находящееся от $x$ на расстоянии Хемминга не более 3.

\section{§4. Некоторые применения кодов в криптографии}

Существует множество работ по синтезу криптографических схем на основе кодов. Примерами таких схем, основанных на линейных кодах, являются системы шифрования Мак-Элиса [20] и Нидеррайтера [25], приведем их краткое описание. 
В основе системы Мак-Элиса лежит некоторый двоичный неприводимый код Гоппы над $\mathrm{GF}\left(2^{m}\right)$ с порождающей матрицей $G_{k \times n}$ длины $n=2^{m}$, размерности $k \geqslant n-t m$, исправляющий $t$ ошибок. Выбираются перестановочная матрица $P_{n \times n}$ и невырожденная матрица $S_{k \times k}$. Использование кодов Гоппы обусловлено существованием быстрого алгоритма декодирования таких кодов.

Далее определяется матрица $(S G P)_{k \times n}$, которая задает линейный код с таким же соотношением $k / n$ и минимальным расстоянием, как у исходного кода Гоппы, и является открытым ключом, как и параметры $n, k, t$.

Шифрование сообщения $u$ длины $k$ заключается в вычислении вектоpa $u \cdot(S G P)_{k \times n}$ и добавлении к нему случайного вектора-ошибки $z$ веса $t$ длины $n$, в результате получаем шифрованное сообщение $x=u \cdot(S G P)_{k \times n}+z$.

Процедура расшифрования сообщения $x$ состоит из выполнения последовательных операций:

1. вычислить $x^{\prime}=x P^{-1}-$ кодовое слово кода Гоппы, где $P^{-1}-$ обратная матрица к $P$;

2. декодировать $x^{\prime}$ с помощью, например, алгоритма Петерсона [27], получив $u^{\prime}$;

3. вычислить $u=u^{\prime} S^{-1}$.

Схема шифрования Нидеррайтера также основана на использовании кодов Гоппы размерности $k \geqslant n-t m$, но задаваемых, в отличие от схемы McEllice, проверочной матрицей $H_{(n-k) \times n}$. Выбираются перестановочная матрица $P_{n \times n}$ и невырожденная матрица $S_{(n-k) \times(n-k)}$. Параметры $n, k, t$ и матрица $(S H P)_{(n-k) \times n}$ являются открытыми, секретными являются матрицы $P, S$ и структура кода Гоппы. Процедуры шифрования и расшифрования аналогичны процедурам для системы McEllice.

Теория кодирования применяется также при построении хэш-функций. Например, в [14] предлагается новый класс конструкций хэш-функций, которые используют коды над $\mathrm{GF}\left(2^{2}\right)$ размерности $k>\frac{n}{2}$ с минимальным расстоянием $d$. Полученные хэш-функции используют блочный шифр $E$ с длиной блока $m$ бит, где $n=O(m)$, имеют внутреннюю память $n \cdot m$ бит и скорость $2 k / n-1$. Нахождение коллизий для таких хэш-функций требует более $2^{(d-1) m / 2}$ операций шифрования; поиск прообраза - не менее $2^{(d-1) m}$ операций.

Приводится конструктивное доказательство существования таких хэшфункций:

$$
h\left(M_{i}, H_{i-1}\right)=E_{M_{i}}\left(H_{i-1}\right) \oplus H_{i-1}, i=1,2, \ldots, t,
$$

где $M_{i}-i$-й блок входного сообщения $M, H_{i}-$ хэш-значение длины $m$ бит, $H_{0}$ - начальное значение, что $h$ требует для построения коллизии более 
$2^{(d-1) m / 2}$ операций шифрования и не менее $2^{(d-1) m}$ операций шифрования для построения прообраза.

Теория кодирования используется для построения криптографических булевых функций. Примерами являются работы [18, 19, 35].

В [19] получены верхние и нижние оценки величины $N\left(d, d^{\perp}\right)-$ минимальной длины линейного кода с минимальным расстоянием $d$ и минимальным расстоянием $d^{\perp}$ дуального к нему кода. Для небольших значений $d$ и $d^{\perp}$ определяется $N\left(d, d^{\perp}\right)$ и предлагается порождающая матрица оптимального линейного кода. Проблема, решаемая в [19], тесно связана с методом построения криптографических функций, который предложил Куросава.

В [35] показано, что если для функции $f\left(x_{1}, x_{2}, \ldots, x_{n}\right)=$ $=\left[y_{1}, y_{2}, \ldots, y_{n-k}\right], f: \mathbb{F}_{q}^{n} \rightarrow \mathbb{F}_{q}^{n-k}$ выполнено условие

$$
\left\{f^{-1}\left(y_{1}, y_{2}, \ldots, y_{n-k}\right): \forall\left(y_{1}, y_{2}, \ldots, y_{n-k}\right) \in \mathbb{F}_{q}^{n-k}\right\}=e+C
$$

где $C$ - систематический $q$-ичный $(n, k)$-код, $e-$ любой элемент множества $E$ всех $q^{n-k}$ наборов длины $n$ с фиксированными 0 позициями, соответствующими информационным символам кода $C$, то $t=d^{\perp}-1$ - максимальное значение, для которого функция $f$ является $t$-устойчивой, где $d^{\perp}-$ минимальное кодовое расстояние дуального кода $C^{\perp}$.

Теорию кодирования можно применять при оценивании нелинейности булевой функции. В [18] предлагается заменить для $n \geqslant 10$ трудоемкое нахождение спектра нелинейности $\left(\delta_{0}, \delta_{1}, \ldots, \delta_{n}\right)$ булевой функции $f\left(x_{1}, x_{2}, \ldots, x_{n}\right)$, где $\delta_{i}-$ нормированное расстояние Хемминга от функции $f$ до ближайшего кодового слова кода Рида-Мюллера $i$-го порядка длины $2^{n}$, поиском похожей характеристики $\left(\tilde{\delta}_{0}, \tilde{\delta}_{1}, \ldots, \tilde{\delta}_{n}\right)$, где $\tilde{\delta}_{i}-$ расстояние до кодового слова, являющегося результатом применения предложенного в [30] алгоритма декодирования кода Рида-Мюллера $i$-го порядка длины $2^{n}$ к таблице истинности функции $f$.

Нередко теория кодов применяется к решению криптографических задач сведением их к решению задачи декодирования, как, например, в $[10,11$, $17,21,22]$.

В [10] и [11] сообщается о новых методах быстрых корреляционных атак на потоковые шифры. Эти методы основаны на технике построения и декодирования турбо- и сверточных кодов. Пусть $\mathcal{L}-$ множество выходных последовательностей двоичного регистра сдвига длины $l$ с линейной обратной 
связью. Очевидно, что $|\mathcal{L}|=2^{l}$ и что для фиксированного $N$ множество усеченных последовательностей $\left\{u=\left(u_{1}, u_{2}, \ldots, u_{N}\right) \mid u \in \mathcal{L}\right\}$ длины $N$ образует линейный $[N, l]$-код $\mathcal{C}$. Пусть последовательность $u=\left(u_{1}, u_{2}, \ldots, u_{N}\right)$ подается на вход двоичного симметричного канала с вероятностью ошибки $p$, $p<0.5$, а на его выходе появляется последовательность $z=\left(z_{1}, z_{2}, \ldots, z_{N}\right)$, т. е. $p=\mathbf{P}\left\{u_{n} \neq z_{n}\right\}$ для любого $n \in\{1,2, \ldots, N\}$.

Задача нахождения кодового слова $\left(u_{1}, u_{2}, \ldots, u_{N}\right) \in \mathcal{C}$ по сообщению $\left(z_{1}, z_{2}, \ldots, z_{N}\right)$ на выходе двоичного симметричного канала связи с вероятностью ошибки $p$ представляет собой пример криптографической задачи (если считать, например, что $u$ - секретная последовательность, а искажения соответствуют ее изменению при прохождении через функцию усложнения).

В [21] описан алгоритм декодирования зашумленной выходной последовательности линейного регистра сдвига с обратной связью, в котором используются апостериорные вероятности каждого символа полученной последовательности; в алгоритме, описанном в [11], используются сверточные коды и метод декодирования Витерби небольших отрезков последовательности. Алгоритм, предложенный в [10], комбинирует методы [21] и [11].

В [17] исследуется задача нахождения сообщения $s \in \mathrm{GF}(2)^{N}$ по полученному зашумленному вектору наблюдений $r \in \mathrm{GF}(2)^{M}$ и двоичной матрице $A$ размера $M \times N$

$$
(A s+n) \bmod 2=r
$$

где $A s-$ кодированное сообщение, $n \in \mathrm{GF}(2)^{M}-$ вектор ошибок.

Похожая задача возникает при анализе корреляционных атак на потоковые шифры, если, например, $s$ - начальное состояние регистра сдвига, $A-$ матрица, связывающая выходной бит регистра с начальным состоянием $s$. В [17] предложен алгоритм нахождения $s$, эмпирическая производительность которого сравнима или даже превосходит (по утверждению автора) производительности кодов Рида-Мюллера и БЧХ; этот алгоритм мог бы заменить алгоритм В Мейера и Стефелбаха, так как его сложность близка к теоретически возможной сложности алгоритма В.

В диссертации [6] можно более подробно ознакомиться с различными применениями теории кодирования, в том числе с применениями в области генерации псевдослучайных последовательностей с помощью эллиптических кривых.

Автор признателен А. М. Зубкову за полезные обсуждения в ходе работы над статьей. 


\section{Список литературы}

1. Agrell E., Vardy A., Zeger K. Upper bounds for constant-weight codes // IEEE Trans. Inf. Theory. - 2000. - V. 46. № 7. - P. 2373-2395.

2. Alfers D., Dinges H. A normal approximation for Beta and Gamma tail probabilities // Z. Wahrscheinlichkeitstheorie verw. Gebiete. - 1984. № 65. - P. 399-420.

3. Bocharova I., Hug F., Johannesson R., Kudryashov B.D. On wieght enumerators and MacWilliams identity for convolutional codes // Theor. Informatics and Appl. - ITA. - 2010.

4. Caragiu M. On a class of constant weight codes // Electr. J. Combinatorics. 1996. - V. 3.

5. Ding C., Yang J. Hamming weights in irreducible cyclic codes // arxiv:1108.3887 - 2011. - P. 1-15.

6. Doumen J. M. Some Applications of Coding Theory in Cryptography // $\mathrm{PhD}$ thesis, Eindhoven Univ. technology. - 2003.

7. Gupta M. K. On some linear codes over $Z_{2^{s}}-1999 .-\mathrm{PhD}$ Diss. Indian Inst. Technology, Kanpur.

8. Hammons A., Kumar P.V., Calderbank A.R., Sloane N.J.A., Sole P. The $Z_{4}$ linearity of Kerdock, Preparata, Goethals and related codes // IEEE Trans. Inform. Theory. - 1994. - V. 40. - P. 301-319.

9. Helleseth T. Codes over $Z_{4}$. // Lect. Notes Comput. Sci. - 2001. - V. 2122. P. 47-55.

10. Johansson T., Jonsson F. Fast correlation attacks based on turbo code techniques // Lect. Notes Comput. Sci. - 1999. - V. 1666. - P. 181-197.

11. Johansson T., Jonsson F. Improved fast correlation attacks on stream ciphers via convolutional codes // Lect. Notes Comput. Sci. - 1999. - V. 1592. P. 347-362.

12. Jurrius R., Pellikaan R. Codes, arrangements and weight enumerators // Dept. of Math. and Comput. Sci., Coding and Crypto Group, The Netherlands. 2009.

13. Kerdock A. M. A class of low-rate nonlinear binary code // Inform. Contr. 1972. - V. 20. - P. 182-187.

14. Knudsen L., Preneel B. Hash functions based on block ciphers and quaternary codes // ASIACRYPT 1996. Lect. Notes Comput. Sci. - 1996. - V. 1163. P. 77-90.

15. Laywine C.F., Mullen G.L. Discrete mathematics using Latin squares. Wiley, 1998. $-326 \mathrm{p}$. 
16. Linial N., Samorodnitsky A. Linear codes and character sums // Combinatorica. - 2002. - P. 497-522.

17. MacKay D.J. A free energy minimization framework for inference problems in modulo 2 arithmetic // Lect. Notes Comput. Sci. - 1995. - V. 1008. - P. 179-195.

18. Massey J. L. Some applications of coding theory in cryptography // Codes and cyphers: cryptography and coding. - 1995. - V. 4. - P. 33-47.

19. Matsumoto R., Kurosawa K. et al. Primal-dual distance bounds of linear codes with application to cryptography // Inf. Theory. - 2006. - V. 52. № 9. P. 4251-4256.

20. McEliece R. J. A public-key cryptosystem based on algebraic coding theory // DSN Progress Report. - 1978. - P. 42-44.

21. Meier, W., Staffelbach, O. Fast correlation attacks on stream ciphers // Lect. Notes Comput. Sci. - 1988. - V. 330. - P. 301-314.

22. Meier $W$., Staffelbach $O$. Fast correlation attacks on certain stream ciphers // J. Cryptology. - 1989. - V. 1. - P. 159-176.

23. Montemanni R., Smith D. H. Heuristic construction of constant weight binary codes // Techn. Rep. No. IDSIA-12-07.

24. Navon M., Samorodnitsky A. Linear programming bounds for codes via a covering argument // Discr. Comput. Geometry. - 2009. - V. 41. № 2. P. 199-207.

25. Niederreiter $H$. Knapsack-type cryptosystems and algebraic coding theory // Пробл. управл. и теории инф. - 1986. - Т. 15. № 2. - С. 159-166.

26. Perry P.N., Fossorier M.P. The average value for the probability of an undetected error // IEEE Trans. Inf. Theory. - 2008. - V. 54. № 5. P. 2372-2375.

27. Patterson N.J. The algeraic dedoding of Goppa codes // IEEE Trans. Inf. Theory. - 1975. - V. 21. - P. 203-207.

28. Preparata F. P. A class of optimum nonlinear double-error correcting codes // Inform. Contr. - 1968. - V. 13. - P. 378-400.

29. Rains E. M. Optimal self-dual codes over $\mathbb{Z}_{4} / /$ Discrete Math. - 1999. V. 203. - P. 215-228.

30. Reed I. S. A class of multiple-error-correcting codes and the decoding scheme // IRE Trans. Inform. Theory. - 1954. - P. 38-49.

31. Sharma A., Bakshi G.K. The weight distribution of some irreducible cyclic codes // Finite Fields and Their Appl. - 2012. - V. 18. № 1. - P. 144-159.

32. Shiromoto $K$. The weight enumerator of linear codes over $G F\left(q^{m}\right)$ having generator matrix over $G F(q)$ // Des., Codes and Cryptography. - 1999. V. 16. № 1. - P. 87-92. 
33. Siap I. The complete weight enumerator for codes over $\mathcal{M}_{n \times s}\left(\mathbb{F}_{q}\right) / /$ IMA Int. Conf. 2001. - P. 20-26.

34. Smith D. H., Hughes L. A., Perkins S. A new table of constant weight codes of length greater than 28 // J. Combinatorics. - 2006. - V. 13.

35. Stinson D. R., Massey J. L. An infinite class of counterexamples to a conjecture concerning non-linear resilient functions // J. Cryptology. - 1995. - V. 8. №3. - P. 167-173.

36. Справочник по специальным функциям (под ред. М. Абрамовица, И. Стиган). - М.: Наука, 1979. - 831 с.

37. Блейхут P. Теория и практика кодов, контролирующих ошибки. - М.: Мир, 1986.

38. Зиновьев В.А., Хеллесет T. О весовых спектрах сдвигов кодов типа Геталса // Проблемы передачи информации. - 2004. - Т. 40. № 2. C. 19-36.

39. Зубков А.М., Серов А.А. Оценки числа булевых функций, имеющих аффинные приближения заданной точности // Дискретная математика. 2010. - T. 22. № 4. - С. 3-19.

40. Зубков А.М., Серов А.А. Полное доказательство универсальных неравенств для функции распределения биномиального закона // Теор. вероятн. и примен. - 2012. - Т. 57. № 3. - С. 597-602.

41. Ллойд С. П. Бинарное блочное кодирование // Кибернетический сб. - М: Изд-во иностр лит., 1960. - Вып. 1. - С. 206-226.

42. Мак-Вильямс Ф.Дж., Слоэн Н.Дж.А. Теория кодов, исправляющих ошибки. - М.: Связь, 1979.

43. Серов А.А. Оценки распределений расстояний от случайной булевой функции до аффинных и квадратичных функций. Кандидатская диссертация. - М.: МИАН, 2011. - 87 с.

44. Серов А. А. Оценки числа булевых функций, имеющих квадратичные приближения заданной точности // Дискретная математика. - 2012. - Т. 24. № 3. - C. 90-107.

45. Сидельников В. М. Теория кодирования. - М.: ФИЗМАТЛИТ, 2008. - 324 с.

46. Сидельников В.М. О спектре весов двоичных кодов Боуза-ЧоудхуриХоквингема // Проблемы передачи информации. - 1971. - Т. 7. Вып. 1. C. $14-22$.

47. Феллер В. Введение в теорию вероятностей и ее приложения, т.1. - М. : Мир, 1984.

48. Шапиро Г. С, Злотник Д. Л. К математической теории кодов с исправлением ошибок // Кибернетический сб. - М.: Изд-во иностр. лит., 1962. Вып. 5. - С. 7-32. 